\title{
Fundamental Limits to Coherent Photon Generation with Solid-State Atomlike Transitions
}

\author{
Z. X. Koong $\odot,{ }^{1,}{ }^{*}$ D. Scerri, ${ }^{1}$ M. Rambach, ${ }^{1}$ T. S. Santana, ${ }^{2}$ S. I. Park, ${ }^{3}$ \\ J.D. Song, ${ }^{3}$ E. M. Gauger, ${ }^{1}$ and B. D. Gerardot ${ }^{1, \dagger}$ \\ ${ }^{1}$ SUPA, Institute of Photonics and Quantum Sciences, Heriot-Watt University, \\ Edinburgh EH14 4AS, Scotland, United Kingdom \\ ${ }^{2}$ Departamento de Física, Universidade Federal de Sergipe, Sergipe, 49100-000, Brazil \\ ${ }^{3}$ Center for Opto-Electronic Materials and Devices Research, Korea Institute of Science and Technology, \\ Seoul 02792, Republic of Korea
}

(Received 11 April 2019; revised manuscript received 19 July 2019; published 16 October 2019)

\begin{abstract}
Coherent generation of indistinguishable single photons is crucial for many quantum communication and processing protocols. Solid-state realizations of two-level atomic transitions or three-level spin- $\Lambda$ systems offer significant advantages over their atomic counterparts for this purpose, albeit decoherence can arise due to environmental couplings. One popular approach to mitigate dephasing is to operate in the weak-excitation limit, where the excited-state population is minimal and coherently scattered photons dominate over incoherent emission. Here we probe the coherence of photons produced using two-level and spin- $\Lambda$ solid-state systems. We observe that the coupling of the atomiclike transitions to the vibronic transitions of the crystal lattice is independent of the driving strength, even for detuned excitation using the spin- $\Lambda$ configuration. We apply a polaron master equation to capture the non-Markovian dynamics of the vibrational manifolds. These results provide insight into the fundamental limitations to photon coherence from solid-state quantum emitters.
\end{abstract}

DOI: 10.1103/PhysRevLett.123.167402

Solid-state quantum emitters can mimic the behavior of few-level atomic systems. Two-level optical transitions can be driven resonantly for coherent manipulation [1-3], which can be used to generate transform-limited single photons [4] with a high degree of indistinguishability from single $[5,6]$ or multiple emitters $[7,8]$. Coherent excitation and control can be extended to solid-state three-level spin$\Lambda$ systems, which enables spin initialization, manipulation, and readout [9-11] as well as spin-photon entanglement [12-14] and indistinguishable single photon generation with tunable temporal and spectral properties [15-20]. These advances can be applied to quantum information applications, for example, distribution of entanglement among independent quantum nodes [21-25] or multiphoton boson sampling [26,27].

At the heart of such quantum applications are photonphoton interactions, achieved when two single-photon wave packets interfere at a beam splitter [28]. Maximum interference visibility demands both coherent and indistinguishable photon wave packets. However, interactions

Published by the American Physical Society under the terms of the Creative Commons Attribution 4.0 International license. Further distribution of this work must maintain attribution to the author(s) and the published article's title, journal citation, and DOI. between the atomlike eigenstates and the solid-state environment can degrade the coherence and indistinguishability of even coherently generated photons. The most prominent mechanisms involve charge [4,29] and spin $[4,11]$ fluctuations and interactions with acoustic phonons [30-40]. One popular approach to mitigate these effects is to use weak coherent excitation. In the weak-excitation regime of a transform-limited optical transition, coherent scattering dominates over incoherent (spontaneous) emission due to minimal excited-state population [41-48]. Likewise, spin-flip Raman scattered photons from solidstate spin- $\Lambda$ systems produce highly coherent photons; in the atomic picture such photons have coherence determined solely by the excitation source and ground (spin) state dephasing [15-20].

In this Letter, we experimentally test the assumption that detrimental interactions with longitudinal acoustic phonons can be minimized by suppressing the excitedstate population in the weak-driving limit. We probe both two-level atomiclike transitions and spin- $\Lambda$ systems in a prototypical solid-state quantum emitter: a charge tunable semiconductor quantum dot (QD). We demonstrate experimentally that the vibrational environment-intrinsic to all solid-state emitters-imposes a fundamental limit on the coherence and indistinguishability of resonantly generated photons from a two-level quantum emitter, independent of driving strength or excited-state population, as 
recently predicted [49]. We proceed to show that this limit equally affects spin-flip Raman scattered photons, contrary to expectations [15-20].

Using a non-Markovian master equation model, we quantitatively explain the coherence as a function of driving strength for both the two-level and spin- $\Lambda$ systems, and we interpret the nonvanishing fraction of incoherently scattered photons as attributable to the vibrational manifolds dressing both the excited and the ground state, and thus also affecting the optical dipole operator (see Fig. 1), even in the absence of excited electronic population. Our results imply that spectral filtering is necessary for perfectly indistinguishable photons, even in the weak-driving and Raman-detuned regimes. This introduces a probabilistic element, hindering the use of solid-state emitters in deterministic single-shot protocols [50-52], even in optimized solid-state phononic or photonic structures [38-40]. Protocols embracing probabilistic operation [53,54], or where the detection of a photon heralds success [21-25], provide mitigation but this limitation remains detrimental.

Figure 1(a) graphically illustrates the electron-phonon coupling, where the charge state of the QD is dressed by

(a)
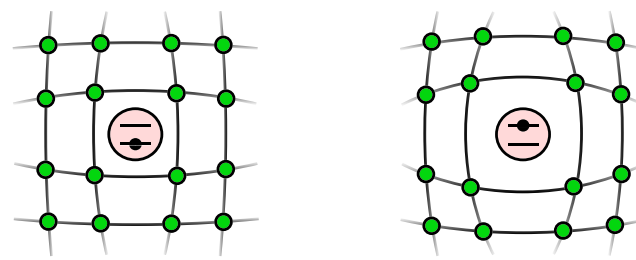

(b)
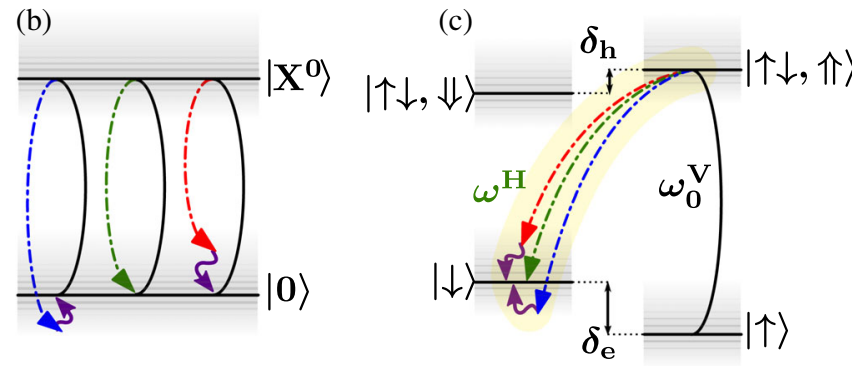

FIG. 1. A quantum dot coupled to vibrational modes. (a) The equilibrium position of crystal lattice ions depends on the charge state of the quantum dot (schematic illustration) due to the deformation potential electron-phonon interaction. (b) Energy level schematic for the neutral exciton $\left(X^{0}\right)$ in the weak-driving regime, showing elastic scattering from the excited state (green) and the inelastic Stokes (red) or anti-Stokes (blue) scattering due to the electronic relaxation from the excited-state to the groundstate vibrational manifold. (c) Spin- $\Lambda$ energy level structure for the negatively charged exciton $\left(X^{1-}\right)$ in the weak-driving regime, showing the inelastic zero-phonon (green) and stokes (red) and anti-stokes (blue) scatterings for the Raman spin-flip transition (yellow). The scattering from the spin-preserving transition is not shown. Single (double) arrows represent electron (heavy-hole) spin states. The energy separation between the Zeeman split ground (excited) states is given by $\delta_{e}\left(\delta_{h}\right)$. lattice displacements. In the strong-driving regime, this interaction is sometimes modeled using a Markovian weakcoupling approach, which correctly captures excitationinduced dephasing [55] and phonon-induced Rabi frequency renormalization [56]. However, the standard weak-coupling approach generally fails to adequately resolve the electron-phonon interaction, and this is particularly evident in the weak-driving regime: here it becomes necessary to treat the electron lattice interaction in terms of phonon-dressed electronic states, so-called polaron quasiparticles. This leads to intrinsically nonMarkovian dynamics for the evolution of the QD charge state, which is also reflected in its optical properties. In the Supplemental Material [57], we extend the non-Markovian polaron model for the neutral exciton [64] to account for the vibrational effects on the charged exciton (which in our case reduces to a spin- $\Lambda$ system). To briefly summarize here, we begin with the canonical Lang-Firsov transformation [65] applied to our Hamiltonian prior to solving its dynamics. In this frame, the original electron-phonon coupling term disappears from the effective Hamiltonian [see Eqs. (20)-(22) in the Supplemental Material [57]]. Instead, we are left with a weaker remnant vibrational coupling term, which we proceed to treat perturbatively. Importantly, both ground and excited states now possess vibrational manifolds, in analogy to the Franck-Condon model [66].

We experimentally probe effective two-level optical transitions and spin- $\Lambda$ systems using the neutral $\left(X^{0}\right)$ and negatively charged $\left(X^{1-}\right)$ excitons, respectively, from a charge tunable QD device [47] at a temperature of $4 \mathrm{~K}$. For $X^{0}$, we resonantly excite and collect from just one of the fine-structure split peaks using orthogonal linear polarizers to suppress the scattered laser background. For $X^{1-}$, we apply an in plane magnetic field (Voigt geometry) of $4 \mathrm{~T}$ to mix the spin states and create "diagonal" spin-flipping transitions (e.g., $|\downarrow\rangle \leftrightarrow|\uparrow \downarrow, \Uparrow\rangle$ ) with horizontal polarization $\left(\omega^{H}\right)$ and equal oscillator strength to the spinconserving transitions (e.g., $|\uparrow\rangle \leftrightarrow|\uparrow \downarrow, \Uparrow\rangle$ ) with vertical polarization $\left(\omega_{0}^{V}\right)$. Here, the single (double) arrows refer to electron (heavy-hole) spin states. We resonantly excite the spin-conserving transition with $\omega_{0}^{V}$ and collect $\omega^{H}$ from the spin-flipping transition, as shown in Fig. 1(c). Photon spectra are characterized by a $\sim 30 \mu \mathrm{eV}$ resolution spectrometer and a $\sim 0.1 \mu \mathrm{eV}$ resolution scanning Fabry-Perot interferometer. A Hanbury Brown-Twiss interferometer and an unbalanced Mach-Zehnder interferometer (with an interferometric delay of $49.7 \mathrm{~ns}$ ) are used to characterize the intensity correlation $g^{(2)}(\tau)$ and postselected, twophoton interference, respectively.

Figures 2(a)-2(f) show the resonantly scattered photon spectra from the $|0\rangle \leftrightarrow\left|X^{0}\right\rangle$ transition at three different driving regimes: $\Omega \approx 0.1 \Omega_{\mathrm{sat}}, \Omega_{\mathrm{sat}}$, and $10 \Omega_{\mathrm{sat}}$, where $\Omega$ $\left(\Omega_{\text {sat }}\right)$ is the Rabi (saturation) frequency. The spectra exhibit identical features as measured by the spectrometer: a 

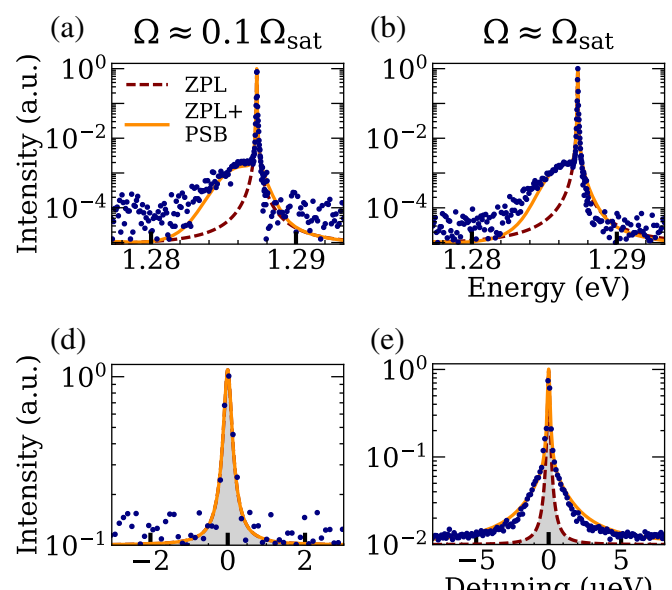

(e)

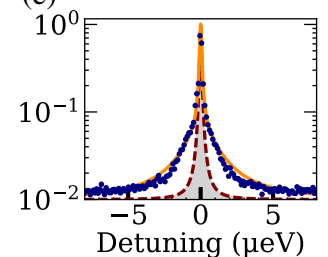

(g)

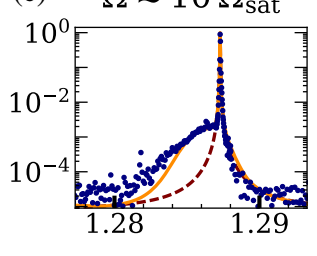

(f)

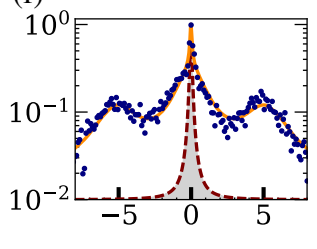

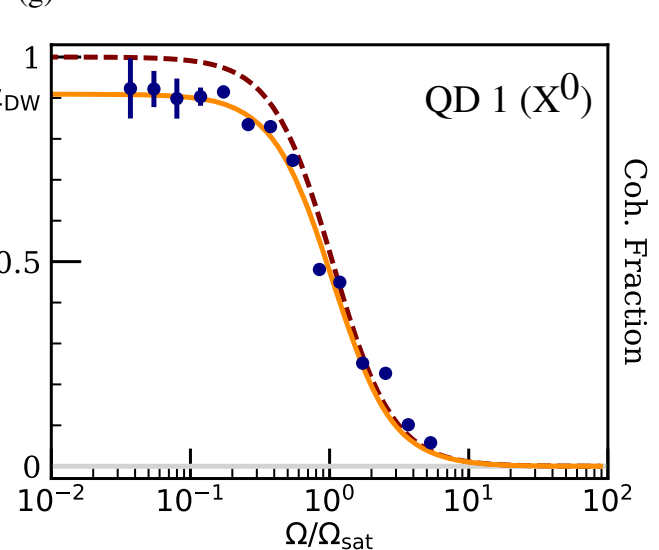

FIG. 2. Resonance fluorescence spectra from a solid-state two-level transition. (a)-(c) Scattered photons at three different Rabi frequencies $\left(\Omega \approx 0.1 \Omega_{\text {sat }}, \Omega_{\text {sat }}, 10 \Omega_{\text {sat }}\right.$ ) show a narrow ZPL accompanied by a broad PSB. The fit is produced by the non-Markovian model, using a super-Ohmic spectral density [68] with system-phonon coupling strength $\alpha=0.03 \mathrm{ps}^{2}$ and frequency cutoff $\omega_{c}=2.2 \mathrm{ps}^{-1}$. (d)-(f) Higher resolution $(\approx 0.1 \mu \mathrm{eV})$ spectra, as a function of the detuning from the ZPL (1.287 eV) show the evolution from a coherent elastic peak at $\Omega \ll \Omega_{\text {sat }}$ to a Mollow triplet at $\Omega \gg \Omega_{\text {sat }}$, matching the behavior of a coherently driven twolevel system. (g) Our theoretical curve (orange) matches the data and confirms that, in the weak-driving regime $\left(\Omega \ll \Omega_{\text {sat }}\right.$ ), the coherence of the transform-limited photons is limited by the branching ratio, given by the Debye-Waller factor, $\alpha_{\mathrm{DW}} \approx 0.91$. Blue data points are experimental data, obtained by computing the ratio of elastic peak [shaded region in (d)-(f)] to total spectrum. The red line shows the theoretical behavior for an atomic two-level system.

narrow zero-phonon line (ZPL, red dashed line) and a broad shoulder near the ZPL— the phonon sideband (PSB). Highresolution spectra of the ZPL reveal a single resolutionlimited elastic peak at $\Omega \approx 0.1 \Omega_{\text {sat }}$ [41-45], the emergence of a broad incoherent spectrum at $\Omega \approx \Omega_{\text {sat }}$, and a Mollow triplet at $\Omega>\Omega_{\text {sat }}$ [1-3]. The orange lines in Figs. 2(d)-2(f) are fits to the experimental data using the theoretical functions as described in Ref. [67] by fixing the lifetime $T_{1}=0.625 \mathrm{~ns}$ and coherence time $T_{2}=2 T_{1}=1.250 \mathrm{~ns}$. The lifetime is independently measured using timeresolved resonance fluorescence [57].

Figures 2(d)-2(f) exhibit textbook atomiclike behavior of resonance fluorescence of a two-level system for the ZPL. On the other hand, the consistent PSB regardless of $\Omega$ in Figs. 2(a)-2(c) reveals a fundamental departure from atomiclike behavior, which can be modeled using the polaron master equation. To tune our microscopic theoretical model to the specific properties of this QD, we have extracted the system-phonon coupling strength and frequency cutoff from the fits to the data in Fig. 2 to be $\alpha=0.03 \mathrm{ps}^{2}$ and $\omega_{c}=2.2 \mathrm{ps}^{-1}$, respectively. The frequency cutoff provides an indication on the size and confinement of the QD [49]. By fitting the experimental data, we extract the ratio of coherent to total (coherent and incoherent) light in the spectrum in the range $0.04 \Omega_{\text {sat }} \leq \Omega \leq 6 \Omega_{\text {sat }}$, as shown in Fig. 2(g), which, at low power, gives the fraction of photons coherently scattered in the ZPL. For an atomic two-level system, this coherent fraction (CF) is determined by [69]

$$
\mathrm{CF}=\frac{T_{2}}{2 T_{1}} \frac{1}{1+\Omega / \Omega_{\mathrm{sat}}},
$$

represented by the dashed red curve in Fig. 2(g). The experimental data depart from Eq. (1) in the weak-excitation regime; to fit the data, we modify the coherent fraction according to

$$
\mathrm{CF}^{\prime}=\alpha_{\mathrm{DW}} \mathrm{CF}
$$

where $\alpha_{\mathrm{DW}}$ is the Debye-Waller coefficient, which quantifies the influence of the vibrational manifold on the nature of scattering process [70] and is equivalent to the square of the Franck-Condon factor $\langle B\rangle[66,71]$, i.e., $\alpha_{\mathrm{DW}}=\langle B\rangle^{2}$ (cf. Refs. [40,49]). Based on the fits of the PSB in the spectra, the theoretical model gives an upper bound of $\alpha_{\text {DW }}=0.91$ as the fraction of maximum coherence in the weak-driving regime.

The fact that the coherent fraction of a two-level system in this $\mathrm{QD}$ is capped at $\alpha_{\mathrm{DW}} \approx 0.91$ in the weak-driving regime shows that a substantial number of emitted photons still interact with the phonon bath, despite the QD population remaining in the ground state throughout. This demonstrates that the phonon sideband is independent of excited-state occupation. Hence, the phonon sideband remains a detrimental effect in exploiting the properties of the photons, namely, the long coherence time of the resonantly scattered photons in the weak-driving regime, contrary to previous claims [41-44].

To verify the spectroscopic observations regarding exciton-phonon coupling and scattered photon coherence, we investigate the two-photon interference. First, we measure the second-order correlation $g^{(2)}(\tau)$ of the scattered photons, which exhibits a suppressed multiphoton emission 
probability of $g^{(2)}(0)=0.046(13)$ at $\Omega \approx 0.1 \Omega_{\text {sat }}$ (see Fig. S2 in the Supplemental Material [57]). Next, we perform Hong-Ou-Mandel (HOM)-type two-photon interference with the unbalanced Mach-Zehnder interferometer. As the two-photon interference visibility $V_{\mathrm{HOM}}(\tau)$ at zerotime delay is solely determined by the detector response time under continuous wave excitation, its maximum value is not indicative of indistinguishability of the photon wave packets [72]. Therefore, we instead consider the coalescence time window $\mathrm{CTW}=\int V_{\mathrm{HOM}}(\tau) d \tau$, given by the shaded area in Figs. 3(a)-3(c), which depends on the full duration of $V_{\mathrm{HOM}}(\tau)$ and is thus independent of detector jitter $[45,48]$. A detailed analysis of CTW for atomic twolevel systems and solid-state nanostructures interacting with a vibrational environment is described in the Supplemental Material [57]. We now investigate the effect of PSB on the CTW as shown in Fig. 3(d). The experimental data points around $\Omega \gtrsim \Omega_{\text {sat }}$ show qualitative agreement with our theoretical model (solid line): the quantitative discrepancy originates from experimental imperfections (e.g., spatial mode overlap or unbalanced splitting ratio of the beam splitter [73]). In the absence of phonons (dashed curve), we find that it differs from our data around $\Omega_{\text {sat }}$ and leads to very different predicted limiting behavior for $\Omega \ll \Omega_{\text {sat }}$. The saturation at lowexcitation power is due to the lifetime limit of the QD: the photon coherence is not solely determined by the laser coherence in this power regime. Thus, the width of the
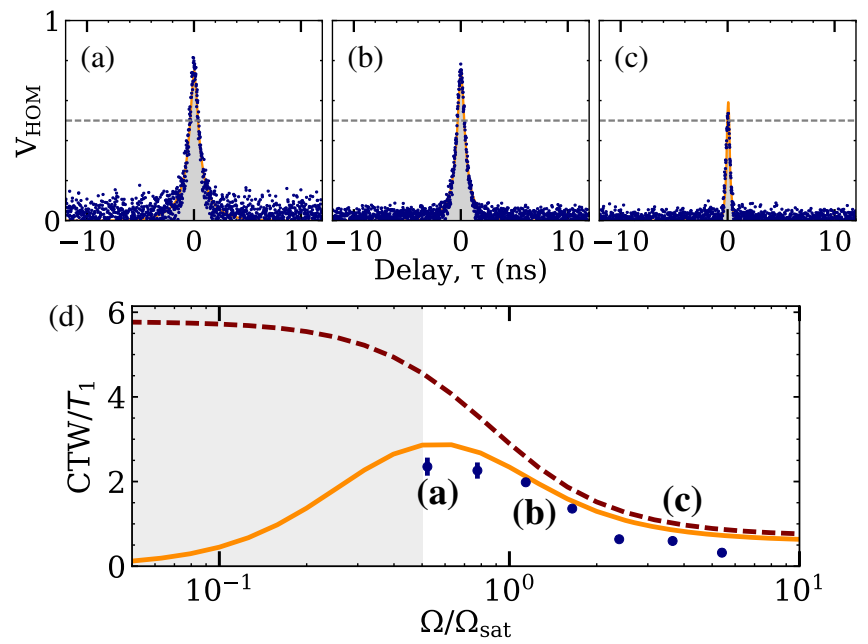

FIG. 3. Two-photon interference between scattered photons from a neutral exciton $X^{0}$ as a function of driving strength. (a)-(c) Two-photon interference visibilities, $V_{\mathrm{HOM}}(\tau)$ at $\Omega \approx$ $0.5 \Omega_{\text {sat }}, \Omega \approx \Omega_{\text {sat }}$, and $\Omega \approx 3 \Omega_{\text {sat }}$, respectively. (d) The coalescence time window normalized by the lifetime of the emitter ( $T_{1}=0.625 \mathrm{~ns}$ ) deduced from the experimental (dots) and theoretical (solid line) CTW as a function of driving Rabi frequency $\Omega$. The dashed line represents the theoretical CTW curve without the contribution from phonons. The shaded region represents the region inaccessible in experiment. visibility dip should converge as $\Omega \rightarrow 0$, resulting in the convergence of the CTW to a finite value, as opposed to increasing indefinitely, even for an ideal continuous wave source $[45,48]$.

Unfortunately, the shaded region $\Omega \lesssim 0.5 \Omega_{\text {sat }}$ is inaccessible in our experimental setup: here the predominantly Rayleigh scattered photons inherit the laser coherence $(\sim 100 \mathrm{kHz})$. This coherence exceeds the interferometric delay in our unbalanced Mach-Zehnder setup, and undesired one-photon interference dominates the measurement $[45,48]$. Nonetheless, the agreement between measured data and the theoretical model for $\Omega \gtrsim \Omega_{\text {sat }}$ supports the validity of the polaron model and justifies the extrapolation into the low-excitation regime. The decreasing CTW is consistent with the reduced $V_{\mathrm{HOM}}(0)$ in Ref. [49], owing to the large separation of timescales between phonon dynamics and average time between scattering events. Our results thus show that the vibrational environment of solid-state emitters degrades the achievable indistinguishability of resonantly scattered photons.

Motivated by the observation that the vibrational environment impacts the ground state of the two-level transition, we proceed to investigate how the phonon interaction affects the spin-flip Raman photon spectrum. The three-level spin- $\Lambda$ system for $X^{1-}$ with an in plane magnetic field is shown in Fig. 1(c).

The vertical transition $|\uparrow\rangle \leftrightarrow|\uparrow \downarrow, \Uparrow\rangle$ is resonantly excited and we collect the scattered Raman photons from the diagonal transition $|\downarrow\rangle \leftrightarrow|\uparrow \downarrow, \Uparrow\rangle$. Figure 4(a) shows the spin-flip Raman photon spectrum in the weak-driving regime, $\Omega \approx 0.1 \Omega_{\text {sat }}$. We observe a narrow ZPL and a broad PSB, independent of $\Omega$, in the low-resolution spectra-similar to the two-level case. The ZPL fraction, given by the ratio of the integrated intensity of the ZPL to the total emission spectrum (ZPL+PSB) is shown in Fig. 4(b) for two QDs, with $\alpha_{\mathrm{DW}}=0.924(4)$ and $\alpha_{\mathrm{DW}}=$ 0.911(4) for QD1 and QD2, respectively. The observation that the ratio of ZPL to PSB of the Raman photons remains constant over 2 orders of magnitude in Rabi frequency leads to the conclusion that the ground spin states are dressed by the vibrational couplings. This contradicts the common consensus that Raman photons are highly coherent and limited only by ground-state spin dephasing [15-20]. We note that the detrimental effect of the electronphonon interaction persists even when there is only negligible excited QD population, so that even Raman red-detuned excitation of the spin- $\Lambda$ system will not eliminate the phonon sideband. Furthermore, we expect the same effect on the wave packet indistinguishability (CTW) as we have discussed for the two-level transition.

To further characterize the coherence of the ZPL of the Raman photons, we measure the spectra from QD2 with the high-resolution Fabry-Perot interferometer. The emergence of a single Gaussian peak with width $\approx 2 \mu \mathrm{eV}$ (FWHM) at $\Omega \approx 0.1 \Omega_{\text {sat }}$ [Fig. 4(c)] and an Autler-Townes doublet at 

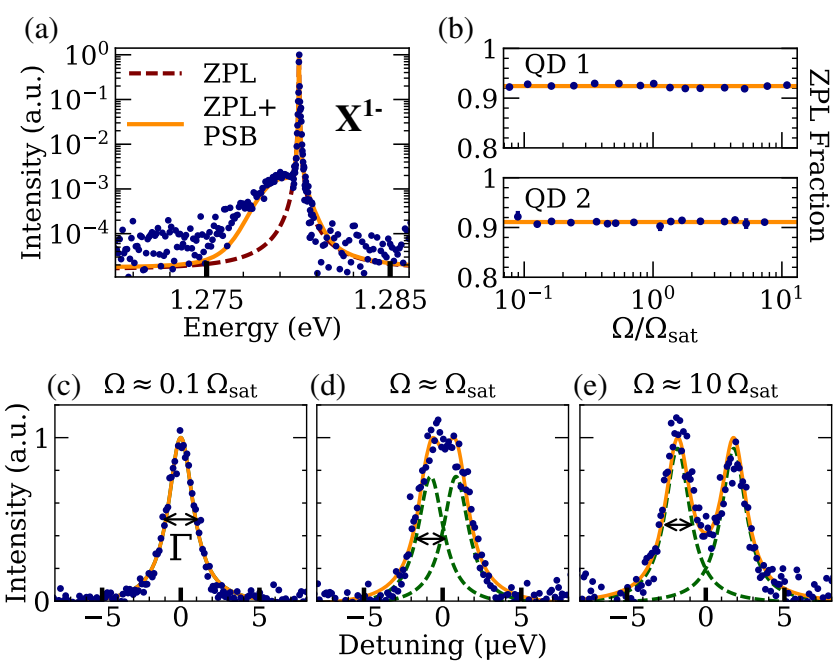

FIG. 4. Spin-flip Raman photon spectra from a solid-state Spin- $\Lambda$ system. (a) Scattered Raman photons from QD1 shows a narrow ZPL and a broad PSB at $\Omega \approx 0.1 \Omega_{\text {sat }}$. The fit is produced by the non-Markovian model using the same set of parameters as in Fig. 2. (b) The ZPL fraction is constant over 2 orders of magnitude in Rabi frequencies. It gives $\alpha_{\mathrm{DW}}=$ 0.924(4) (QD1) and $\alpha_{\mathrm{DW}}=0.911(4)$ (QD2). (c)-(e) Emission spectra of the scattered Raman photons from QD2 at three different Rabi frequencies $\left(\Omega \approx 0.1 \Omega_{\text {sat }}, \Omega_{\text {sat }}, 10 \Omega_{\text {sat }}\right.$ ) show an Autler-Townes splitting at $\Omega \gtrsim \Omega_{\text {sat }}$. Zero detuning corresponds to energy of ZPL at $1.277 \mathrm{eV}$. The broad linewidth $(\Gamma \approx 2 \mu \mathrm{eV})$ originates from the spin ground-state dephasing due to nuclear spin fluctuations.

$\Omega \approx \Omega_{\text {sat }}$ [Figs. 4(d) and 4(e)] confirm the nature of coherent driving in the spin- $\Lambda$ system. As explained in the Supplemental Material [57], the relatively broad linewidth $(\Gamma \approx 2 \mu \mathrm{eV}$ ) of the spin-flip Raman photon is due to the spin ground-state dephasing, which is dominated by the coupling of electron spin to the nuclear spin bath $[18,47]$.

In summary, our experimental and theoretical results contradict the expectation that perfectly coherent photons can be obtained from a solid-state emitter, either by weak resonant driving of a two-level transition or as Raman scattered photons. Instead, we have shown that the solidstate environment, and the associated exciton-phonon interaction, invariably limit the coherence of resonantly scattered photons: a minimum fraction $\alpha_{\mathrm{DW}}$ of photons are scattered incoherently, for any excitation power and scheme. We argue that these phonon-induced effects are due to the relaxation of the phonon bath in the excitonic ground state, explaining why neither the weak-driving nor Raman red-detuned excitation regimes mitigate the interaction. While it is possible to filter the phonon sideband, this introduces a probabilistic element to the success rate of obtaining indistinguishable photons. Furthermore, despite being an intuitive solution, embedding the QD inside a cavity or waveguide for strong Purcell enhancement can only partly reduce the adverse effects of the vibrational environment, and, in the case of a cavity-embedded QD, at the cost of efficiency [40]. Our non-Markovian polaron frame model agrees well with the experimental data for both the two- and three-level solid-state systems, showing that the presence of the vibrational environment impacts the emitter's dynamics even in the low-power, Raman reddetuned regime.

This work was supported by the EPSRC (Grants No. EP/ L015110/1, No. EP/M013472/1, and No. EP/P029892/1), the ERC (Grant No. 725920), and the EU Horizon 2020 research and innovation program under Grant Agreement No. 820423. B. D. G. thanks the Royal Society for a Wolfson Merit Award and the Royal Academy of Engineering for a Chair in Emerging Technology, and E. M. G. acknowledges financial support from the Royal Society of Edinburgh and the Scottish Government. T. S. S. acknowledges PNPD/CAPES for financial support. The authors in K. I.S.T. acknowledge the support from the KIST institutional program and the program of quantum sensor core technology through IITP.

Note added.-Recently, we became aware of related results [74]. We thank A. J. Brash for bringing this to our attention.

*zk49@hw.ac.uk

†b.d.gerardot@hw.ac.uk

[1] G. Wrigge, I. Gerhardt, J. Hwang, G. Zumofen, and V. Sandoghdar, Nat. Phys. 4, 60 (2008).

[2] A. Nick Vamivakas, Y. Zhao, C.-Y. Lu, and M. Atatüre, Nat. Phys. 5, 198 (2009).

[3] E. B. Flagg, A. Muller, J. W. Robertson, S. Founta, D. G. Deppe, M. Xiao, W. Ma, G. J. Salamo, and C. K. Shih, Nat. Phys. 5, 203 (2009).

[4] A. V. Kuhlmann, J. H. Prechtel, J. Houel, A. Ludwig, D. Reuter, A. D. Wieck, and R. J. Warburton, Nat. Commun. 6, 8204 (2015).

[5] X. Ding, Y. He, Z.-C. Duan, N. Gregersen, M.-C. Chen, S. Unsleber, S. Maier, C. Schneider, M. Kamp, S. Höfling, C.-Y. Lu, and J.-W. Pan, Phys. Rev. Lett. 116, 020401 (2016).

[6] N. Somaschi, V. Giesz, L. De Santis, J. C. Loredo, M. P. Almeida, G. Hornecker, S. L. Portalupi, T. Grange, C. Antón, J. Demory, C. Gómez, I. Sagnes, N. D. LanzillottiKimura, A. Lemaítre, A. Auffeves, A. G. White, L. Lanco, and P. Senellart, Nat. Photonics 10, 340 (2016).

[7] R. Lettow, Y. L. A. Rezus, A. Renn, G. Zumofen, E. Ikonen, S. Götzinger, and V. Sandoghdar, Phys. Rev. Lett. 104, 123605 (2010).

[8] H. Bernien, L. Childress, L. Robledo, M. Markham, D. Twitchen, and R. Hanson, Phys. Rev. Lett. 108, 043604 (2012).

[9] D. Press, T. D. Ladd, B. Zhang, and Y. Yamamoto, Nature (London) 456, 218 (2008).

[10] C. G. Yale, B. B. Buckley, D. J. Christle, G. Burkard, F. J. Heremans, L. C. Bassett, and D. D. Awschalom, Proc. Natl. Acad. Sci. U.S.A. 110, 7595 (2013). 
[11] A. Bechtold, D. Rauch, F. Li, T. Simmet, P.-L. Ardelt, A. Regler, K. Müller, N. A. Sinitsyn, and J. J. Finley, Nat. Phys. 11, 1005 (2015).

[12] E. Togan, Y. Chu, A. S. Trifonov, L. Jiang, J. Maze, L. Childress, M. V. G. Dutt, A. S. Sørensen, P. R. Hemmer, A. S. Zibrov, and M. D. Lukin, Nature (London) 466, 730 (2010).

[13] W. B. Gao, P. Fallahi, E. Togan, J. Miguel-Sanchez, and A. Imamoğlu, Nature (London) 491, 426 (2012).

[14] K. De Greve, L. Yu, P. L. McMahon, J. S. Pelc, C. M. Natarajan, N. Y. Kim, E. Abe, S. Maier, C. Schneider, M. Kamp, S. Höfling, R. H. Hadfield, A. Forchel, M. M. Fejer, and Y. Yamamoto, Nature (London) 491, 421 (2012).

[15] C. Santori, D. Fattal, K.-M. C. Fu, P. E. Barclay, and R. G. Beausoleil, New J. Phys. 11, 123009 (2009).

[16] G. Fernandez, T. Volz, R. Desbuquois, A. Badolato, and A. Imamoğlu, Phys. Rev. Lett. 103, 087406 (2009).

[17] Y. He, Y.-M. He, Y.-J. Wei, X. Jiang, M.-C. Chen, F.-L. Xiong, Y. Zhao, C. Schneider, M. Kamp, S. Höfling, C.-Y. Lu, and J.-W. Pan, Phys. Rev. Lett. 111, 237403 (2013).

[18] Z. Sun, A. Delteil, S. Faelt, and A. Imamoğlu, Phys. Rev. B 93, 241302(R) (2016).

[19] L. Béguin, J.-P. Jahn, J. Wolters, M. Reindl, Y. Huo, R. Trotta, A. Rastelli, F. Ding, O. G. Schmidt, P. Treutlein, and R. J. Warburton, Phys. Rev. B 97, 205304 (2018).

[20] B. C. Pursley, S. G. Carter, M. K. Yakes, A. S. Bracker, and D. Gammon, Nat. Commun. 9, 115 (2018).

[21] C. Cabrillo, J. I. Cirac, P. García-Fernández, and P. Zoller, Phys. Rev. A 59, 1025 (1999).

[22] S. D. Barrett and P. Kok, Phys. Rev. A 71, 060310(R) (2005).

[23] H. Bernien, B. Hensen, W. Pfaff, G. Koolstra, M. S. Blok, L. Robledo, T. H. Taminiau, M. Markham, D. J. Twitchen, L. Childress, and R. Hanson, Nature (London) 497, 86 (2013).

[24] A. Delteil, Z. Sun, W.-b. Gao, E. Togan, S. Faelt, and A. Imamoğlu, Nat. Phys. 12, 218 (2016).

[25] R. Stockill, M. J. Stanley, L. Huthmacher, E. Clarke, M. Hugues, A. J. Miller, C. Matthiesen, C. Le Gall, and M. Atatüre, Phys. Rev. Lett. 119, 010503 (2017).

[26] H. Wang, Y. He, Y.-H. Li, Z.-E. Su, B. Li, H.-L. Huang, X. Ding, M.-C. Chen, C. Liu, J. Qin, J.-P. Li, Y.-M. He, C. Schneider, M. Kamp, C.-Z. Peng, S. Höfling, C.-Y. Lu, and J.-W. Pan, Nat. Photonics 11, 361 (2017).

[27] J. C. Loredo, M. A. Broome, P. Hilaire, O. Gazzano, I. Sagnes, A. Lemaitre, M. P. Almeida, P. Senellart, and A. G. White, Phys. Rev. Lett. 118, 130503 (2017).

[28] C. K. Hong, Z. Y. Ou, and L. Mandel, Phys. Rev. Lett. 59, 2044 (1987).

[29] J. Houel, A. V. Kuhlmann, L. Greuter, F. Xue, M. Poggio, B. D. Gerardot, P. A. Dalgarno, A. Badolato, P. M. Petroff, A. Ludwig, D. Reuter, A. D. Wieck, and R. J. Warburton, Phys. Rev. Lett. 108, 107401 (2012).

[30] S. Hameau, Y. Guldner, O. Verzelen, R. Ferreira, G. Bastard, J. Zeman, A. Lemaître, and J. M. Gérard, Phys. Rev. Lett. 83, 4152 (1999).

[31] J. Seebeck, T. R. Nielsen, P. Gartner, and F. Jahnke, Phys. Rev. B 71, 125327 (2005).

[32] A. Vagov, V. M. Axt, T. Kuhn, W. Langbein, P. Borri, and U. Woggon, Phys. Rev. B 70, 201305(R) (2004).
[33] P. Borri, W. Langbein, U. Woggon, V. Stavarache, D. Reuter, and A. D. Wieck, Phys. Rev. B 71, 115328 (2005).

[34] P. Kaer, P. Lodahl, A.-P. Jauho, and J. Mork, Phys. Rev. B 87, 081308(R) (2013).

[35] K. Roy-Choudhury and S. Hughes, Opt. Lett. 40, 1838 (2015).

[36] A. Thoma, P. Schnauber, M. Gschrey, M. Seifried, J. Wolters, J.-H. Schulze, A. Strittmatter, S. Rodt, A. Carmele, A. Knorr, T. Heindel, and S. Reitzenstein, Phys. Rev. Lett. 116, 033601 (2016).

[37] A. Reigue, J. Iles-Smith, F. Lux, L. Monniello, M. Bernard, F. Margaillan, A. Lemaitre, A. Martinez, D. P. S. McCutcheon, J. Mørk, R. Hostein, and V. Voliotis, Phys. Rev. Lett. 118, 233602 (2017).

[38] P. Tighineanu, C. L. Dreeßen, C. Flindt, P. Lodahl, and A. S. Sørensen, Phys. Rev. Lett. 120, 257401 (2018).

[39] T. Grange, N. Somaschi, C. Antón, L. De Santis, G. Coppola, V. Giesz, A. Lemaître, I. Sagnes, A. Auffèves, and P. Senellart, Phys. Rev. Lett. 118, 253602 (2017).

[40] J. Iles-Smith, D. P. S. McCutcheon, A. Nazir, and J. Mørk, Nat. Photonics 11, 521 (2017).

[41] H. S. Nguyen, G. Sallen, C. Voisin, P. Roussignol, C. Diederichs, and G. Cassabois, Appl. Phys. Lett. 99, 261904 (2011).

[42] K. Konthasinghe, J. Walker, M. Peiris, C. K. Shih, Y. Yu, M. F. Li, J. F. He, L. J. Wang, H. Q. Ni, Z. C. Niu, and A. Muller, Phys. Rev. B 85, 235315 (2012).

[43] C. Matthiesen, A. N. Vamivakas, and M. Atatüre, Phys. Rev. Lett. 108, 093602 (2012).

[44] C. Matthiesen, M. Geller, C. H. H. Schulte, C. Le Gall, J. Hansom, Z. Li, M. Hugues, E. Clarke, and M. Atatüre, Nat. Commun. 4, 1600 (2013).

[45] R. Proux, M. Maragkou, E. Baudin, C. Voisin, P. Roussignol, and C. Diederichs, Phys. Rev. Lett. 114, 067401 (2015).

[46] C. H. H. Schulte, J. Hansom, A. E. Jones, C. Matthiesen, C. Le Gall, and M. Atatüre, Nature (London) 525, 222 (2015).

[47] R. N. E. Malein, T. S. Santana, J. M. Zajac, A. C. Dada, E. M. Gauger, P. M. Petroff, J. Y. Lim, J. D. Song, and B. D. Gerardot, Phys. Rev. Lett. 116, 257401 (2016).

[48] E. Baudin, R. Proux, M. Maragkou, P. Roussignol, and C. Diederichs, Phys. Rev. A 99, 013842 (2019).

[49] J. Iles-Smith, D. P. S. McCutcheon, J. Mørk, and A. Nazir, Phys. Rev. B 95, 201305(R) (2017).

[50] N. H. Lindner and T. Rudolph, Phys. Rev. Lett. 103, 113602 (2009).

[51] D. Buterakos, E. Barnes, and S. E. Economou, Phys. Rev. X 7, 041023 (2017).

[52] M. Gimeno-Segovia, T. Rudolph, and S.E. Economou, Phys. Rev. Lett. 123, 070501 (2019).

[53] T. Rudolph, APL Photonics 2, 030901 (2017).

[54] D. Scerri, R. N. E. Malein, B. D. Gerardot, and E. M. Gauger, Phys. Rev. A 98, 022318 (2018).

[55] A. J. Ramsay, A. V. Gopal, E. M. Gauger, A. Nazir, B. W. Lovett, A. M. Fox, and M. S. Skolnick, Phys. Rev. Lett. 104, 017402 (2010).

[56] A. J. Ramsay, T. M. Godden, S. J. Boyle, E. M. Gauger, A. Nazir, B. W. Lovett, A. M. Fox, and M. S. Skolnick, Phys. Rev. Lett. 105, 177402 (2010). 
[57] See Supplemental Material at http://link.aps.org/ supplemental/10.1103/PhysRevLett.123.167402 for description of the experimental setup, details on the postselect two-photon interference measurement, coalescence time window, the nuclear spin coupling to Raman photons, and the derivation of the associated polaron master equation for a spin- $\Lambda$ system, which includes Refs. [58-63].

[58] Z. Ficek, T. Asakura, S. Swain, K. Brenner, T. Hänsch, T. Kamiya, F. Krausz, B. Monemar, W. Rhodes, H. Venghaus et al., Quantum Interference and Coherence: Theory and Experiments, Springer Series in Optical Sciences (Springer, New York, 2005).

[59] A. Lebreton, I. Abram, R. Braive, I. Sagnes, I. RobertPhilip, and A. Beveratos, Phys. Rev. A 88, 013801 (2013).

[60] B. Urbaszek, X. Marie, T. Amand, O. Krebs, P. Voisin, P. Malentinsky, A. Högele, and A. Imamoğlu, Rev. Mod. Phys. 85, 79 (2013).

[61] D. Scerri, T. S. Santana, B. D. Gerardot, and E. M. Gauger, Phys. Rev. B 95, 165403 (2017).

[62] K. Roy-Choudhury and S. Hughes, Phys. Rev. B 92, 205406 (2015).

[63] H. Breuer and F. Petruccione, The Theory of Open Quantum Systems (Oxford University Press, Oxford, 2007).

[64] A. Nazir and D. P. S. McCutcheon, J. Phys. Condens. Matter 28, 103002 (2016).
[65] I. Lang and Y. A. Firsov, J. Exp. Theor. Phys. 43, 1301 (1962).

[66] G. Mahan, Many-Particle Physics, Physics of Solids and Liquids (Springer, New York, 2000).

[67] M. O. Scully and M. S. Zubairy, Quantum Optics (Cambridge University Press, Cambridge, England, 1997).

[68] A. J. Leggett, S. Chakravarty, A. T. Dorsey, M. P. A. Fisher, A. Garg, and W. Zwerger, Rev. Mod. Phys. 59, 1 (1987).

[69] C. Cohen-Tannoudji, J. Dupont-Roc, and G. Grynberg, Atom-Photon Interactions: Basic Process and Applications, , 1st ed. (Wiley, New York, 1998), pp. 369-383.

[70] H. J. Lipkin, arXiv:cond-mat/0405023.

[71] The equivalence between $\langle B\rangle$ and the Franck-Condon factor stems from the fact that both quantities emerge as vibrational prefactors when taking the transition probability of the system coupled to the vibrational environment.

[72] T. Legero, T. Wilk, M. Hennrich, G. Rempe, and A. Kuhn, Phys. Rev. Lett. 93, 070503 (2004).

[73] V. Giesz, S. L. Portalupi, T. Grange, C. Antón, L. De Santis, J. Demory, N. Somaschi, I. Sagnes, A. Lemaître, L. Lanco, A. Auffèves, and P. Senellart, Phys. Rev. B 92, 161302(R) (2015).

[74] A. J. Brash, J. Iles-Smith, C. L. Phillips, D. P. S. McCutcheon, J. O'Hara, E. Clarke, B. Royall, J. Mørk, M. S. Skolnick, A. M. Fox, and A. Nazir, following Letter, Phys. Rev. Lett. 123, 167403 (2019). 